\title{
SYNTAXE ET SÉMANTIQUE CHEZ TESNIÈRE
}

Ce qui frappe avant tout dans les Eléments de syntaxe structurale de Lucien Tesnière, c'est leur caractère exhaustif: il s'agit d'une somme, rendant compte potentiellement de tous les phénomènes syntaxiques de toutes les langues. Aucun des autres grands linguistes du $\mathrm{XX}^{\circ}$ siècle ne nous présente une oeuvre comparable, tous, de Hjelmslev à Chomsky, insistent davantage sur des questions de méthode sans en rechercher l'application à tous les problèmes possibles.

Mais cette somme frappe en outre par sa cohérence. Chez un auteur qui part de principes méthodologiques, la cohérence est donnée a priori et ne saurait surprendre, d'autant plus que les faits qui mettraient cette cohérence en défaut peuvent fort bien être passés sous silence. Chez celui qui prétend, suivant le précepte de Descartes, procéder à des "dénombremens entiers", la cohérence est une vertu chèrement acquise et qui ne peut que susciter l'admiration.

C'est cela d'abord qui passionne le lecteur chez Tesnière: autour de quelques concepts simples, utilisés avec rigueur dans tout le livre, toutes les constructions syntaxiques de langues typologiquement très diverses se rangent rigoureusement dans la majestueuse ordonnance du stemma, procédé de représentation universel. Les concepts utilisés par les diverses écoles linguistiques pour classer les catégories et les fonctions retrouvent chacun leur place rationnelle dans cette construction grandiose.

Les deux éléments essentiels en sont la théorie de la dépendance et celle des actants et circonstants. Grâce à la première, tous les éléments signifiants de n'importe quelle phrase sont mis en rapports univoques les uns.avec les autres, si bien que rien n'échappe. Grâce à la seconde (complétée par la notion de translation), la théorie intègre les fonctions traditionnelles de sujet, objet etc., et éclaire les rapports entre les catégories de mots (parties du discours) et leurs fonctions.

Il est pourtant permis de se demander, en revoyant le livre de Tesnière d'un oeil critique, si la cohérence est aussi grande qu'il apparaît d'abord. Ses deux aspects: la théorie de la dépendance et celle des actants et circonstants, sont-ils bien liés entre eux et découlent-ils logiquement l'un de l'autre? Nous pensons qu'une étude attentive permet de découvrir une faille entre ces deux éléments fondamentaux du système, mais que l'exploration même de cette faille est riche d'enseignements et ne rend que plus évidente la fécondité de l'oeuvre. 
La notion de dépendance est introduite dès le deuxième chapitre des Eléments (p. 13): "Les connexions structurales établissent entre les mots des rapports de dépendance. Chaque connexion unit en principe un terme supérieur à un terme inférieur. Le terme supérieur reçoit le nom de régissant. Le terme inférieur reçoit le nom de subordonné. Ainsi dans la phrase Alfred parle, parle est le régissant et Alfred le subordonné".

Dans la suite du livre cette notion est utilisée systématiquement et appliquée à toutes les connexions possibles. C'est elle qui justifie la construction du stemma, qui permet une représentation particulièrement harmonieuse et efficace de n'importe quelle phrase. Mais c'est finalement cette utilité pragmatique qui justifie a posteriori, dans chaque connexion, l'attribution à l'un des termes du rôle de régissant et à l'autre du rôle de subordonné.

En revanche nous ne trouvons nulle part chez l'auteur de justification théorique de cette répartition. Il n'y a dans tout le livre aucune définition de l'un et l'autre terme, du type: "Le terme régissant est celui qui ...; le terme subordonné est celui qui ...". Là-même où Tesnière oppose avec vigueur sa propre conception du rapport hiérarchique verbe-sujet à la conception traditionnelle du rapport égalitaire sujet-prédicat (p. 102-105), il ne tire argument que des conséquences que peuvent avoir l'une et l'autre conception sur l'économie de la description, mais non sur une définition des deux termes. Il ne discute nulle part non plus la position inverse de la sienne, celle qui subordonne le verbe au sujet, et pourtant ce point de vue est celui de Charles Bally dans sa Linguistique générale et linguistique française (Bally 1932), livre que Tesnière cite fréquemment. La dépendance et le sens dans laquelle elle joue ont en somme chez lui le statut d'une hypothèse intuitivement découverte, qui ne se justifie que par ses conséquences.

On peut chercher à combler cette lacune et s'efforcer de définir ce qu'est dans une connexion le terme supérieur et le terme inférieur. Tesnière nous met sur la voie en notant (p. 14) que "tout subordonné suit le sort de son régissant". Cette remarque, qui n'a pas à ses yeux statut de définition, nous paraît pourtant être implicitement la clé de sa conception. A partir de là on peut définir le terme supérieur comme celui qui assure la liaison de l'ensemble de la connexion avec le contexte où elle s'insère (Sur cette définition, inspirée en partie de Mel'čuk 1964, cf. Garde 1977). Ainsi le verbe est le terme principal de la proposition parce que c'est la forme verbale qui détermine le statut indépendant ou subordonné de celle-ci. On s'aperçoit alors que la détermination de la dépendance est fondée exclusivement sur des considérations intra-linguistiques, à l'exclusion de tout appel à la sémantique, ce qui est conforme aux vues exprimées par Tesnière, qui insiste (p. 40 et passim) sur la "distiction entre le plan structural et le plan sémantique".

La détermination ainsi obtenue, dans chaque connexion, du sens de la dépendance, est nécessaire et suffisante pour la construction du stemma, lequel ne figure rien d'autre que des rapports de régissant à subordonné, donc des rapports verticaux. Tesnière 
lui-même note (p. 15) que si le stemma comprend des traits obliques, c'est que "comme les connexions inférieures peuvent être multiples, on est amené, dans la représentation graphique, à tricher sur les traits de connexion et à en faire des traits obliques". En tout cas, il n'y a aucun trait horizontal. Il n'y a rien non plus, dans l'examen des rapports de dépendance ainsi obtenus, qui incite à placer les divers subordonnés d'un même régissant dans un ordre plutôt que dans un autre. Soit la phrase Hier Alfred a oublié son chapeau (p. 61). Les considérations intra-linguistiques de dépendance nous obligent a considérer les trois éléments hier, Alfred et son chapeau comme subordonnés tous trois au verbe a oublié, et à les inscrire en-dessous de lui dans le stemma. Mais elles ne nous disent pas dans quel ordre doivent être inscrits, sur le plan horizontal, ces trois éléments. Rien, dans les rapports de dépendance, ne nous empêcherait de les faire se succéder selon l'ordre linéaire, soit 1- hier, 2- Alfred, 3- son chapeau, ou selon n'importe quelle autre disposition. Si Tesnière choisit la séquence 1- Alfred, 2-son chapeau, 3- hier, et s'il y voit "l'ordre structural" qu'il oppose à "l'ordre linéaire", c'est pour des raisons qui ne se déduisent en aucune façon des considérations de dépendance, et qui ont leur propre autonomie.

Cette disposition se réfère à un tout autre volet de l'oeuvre. Elle découle du classement que Tesnière établit parmi les différents subordonnés du nœud verbal, en définissant Alfred comme "prime actant", son chapeau comme "second actant" et hier comme "circonstant". Elle nous renvoie donc à la définition de ces notions, qui figure dans le chapitre 48 des Eléments: "Le nœud verbal" (p. 102):

"1- Le nœud verbal [...] exprime tout un petit drame. Comme un drame en effet, il comporte obligatoirement un procès, et le plus souvent des acteurs et des circonstances.

"2- Transposés du plan de la réalité dramatique sur celui de la syntaxe structurale, le procès, les acteurs et les circonstances deviennent respectivement le verbe, les actants et les circonstants. [...]

4- Les actants sont les êtres ou les choses qui, à un titre quelconque et de quelque façon que ce soit, même au titre de simples figurants et de la façon la plus passive, participent au procès. [...]

7- Les circonstants expriment les circonstances: de temps, lieu, manière etc. dans lesquelles se déroule le procès".

Il est facile de voir le changement de critères par rapport à l'introduction des notions précédentes. Les définitions proposées ici (cette fois explicitement) se fondent sur ce qui est exprimé par le nœud verbal, et considère la syntaxe comme la simple transposition d'une réalité dramatique. C'est dire que les critères sont ici sémantiques, et non plus "structuraux"; qu'ils se réfèrent à la réalité extra-linguistique, et non plus aux rapports intra-linguistiques.

Il y a donc dans la théorie de Tesnière deux aspects mutuellement indépendants, qui ne découlent pas logiquement l'un de l'autre. Le stemma, figurant les dépendances, 
ne perdrait rien de sa rigueur si la théorie des actants n'existait pas. Cette dernière, de son côté, pourrait aussi bien être développée dans le cadre d'une grammaire qui ne se fonderait pas sur les dépendances. C'est un peu ce qu'on voit, mutatis mutandis, dans la "grammaire des cas" de Fillmore, avec sa notion de "cas profonds" (sémantiques). Ce n'est pas un hasard si la postérité de Tesnière, en ce qui concerne la grammaire des dépendances, a été le fait de linguistes au sens strict, tandis que sa théorie des actants a été féconde avant tout chez des sémioticiens, notamment en narratologie.

Mais la démarche la plus féconde nous paraît être celle qui consiste, après avoir pris conscience de la différence entre ces deux aspects de la théorie, de les considérer néanmoins ensemble, comme nous y invite l'œuvre de Tesnière, en réfléchissant sur leurs rapports. On peut poser alors quelques antinomies:

1- la dépendance est d'ordre "structural" (syntaxique), la théorie des actants d'ordre sémantique.

2- cela signifie que la dépendance examine des rapports intra-linguistiques, la théorie des actants des rapports extra-linguistiques.

3- il en résulte que la théorie des actants ne s'applique qu'aux éléments ayant un référent extra-linguistique, les "mots pleins" de Tesnière (p. 53). Au contraire, la notion de dépendance s'applique à tous les éléments signifiants, donc à tous les morphèmes, même ceux qui n'ont comme signifiant que des rapports extra-linguistiques: "mots vides" selon Tesnière, et éléments morphologiques du mot, puisque chaque morphème joue un rôle dans l'insertion de l'ensemble auquel il appartient dans son contexte. Pour reprendre un exemple de Tesnière (p. 58), mais contrairement à son avis, quand lat. bona mente fr. bonne-ment, l'univerbation ne change pas les rapports de dépendance entre les deux éléments (mots pleins distincts en latin, morphèmes en français). C'est toujours le second: mente, -ment qui est le régissant, parce que c'est lui qui détermine l'insertion de l'ensemble dans son contexte.

4- dans les rapports de dépendance l'élément principal ("régissant" au sens de Tesnière) est le verbe. Mais les rapports sémantiques s'ordonnent plutôt autour du nom, comme le suggère la théorie de Bally (1932, déjà cité), ou celle d'Otto Jespersen (1933, p. $78 \mathrm{sq}$.), qui distingue des mots de $1^{\circ}$ degré (noms), de $2^{\circ}$ degré (adjectif et verbe) et de $3^{\circ}$ degré (adverbes).

5- Les rapports de dépendance ont un mode d'expression morphologique privilégie, c'est la rection: le terme principal (appelé justement par Tesnière "régissant") détermine la forme du subordonné (cas, préposition etc.). Mais les rapports sémantiques ont aussi un mode d'expression morphologique favori, qui est l'accord: celui-ci, là où il existe, part toujours d'un nom, et affecte toujours des mots (adjectifs, verbes ou autres noms) ayant avec lui des rapports sémantiques d'identité.

Le livre de Tesnière procède à un inventaire extrêmement complet, et qui ne peut guère être dépassé, des rapports de dépendance. Il va moins loin dans celle des rapports proprement sémantiques, puisqu'il ne les envisage que là où précisément ils coïncident 
avec des rapports de description systématique des deux réseaux; celui des rapports de dépendance, mais aussi celui des rapports sémantiques, et des relations entre eux dans langues diverses. Ainsi la "faille" logique que nous avons cru déceler dans le raisonnement de Tesnière ne saurait être considérée comme un défaut, mais au contraire comme une invitation à de plus amples recherches dont le maître de Montpellier aura été l'inspirateur.

\section{Bibliographie}

[Les références données dans le texte sans nom d'auteur renvoient à Tesnière 1959]. Bally, Charles, 1932, Linguistique générale et linguistique française, Genève (cité d'après la $4^{\circ}$ éd., Berne, Francke, 1965).

Garde, Paul, 1977, "Ordre linéaire et dépendance syntaxique. Contribution à une typologie", Bulletin de la société de linguistique de Paris, 72, 1, p. 1-26.

Jespersen, Otto, 1933, Essentials of English Grammar, Londres, Allen and Unwin.

Mel'čuk, I.A., 1964, Avtomatičeskij sintaksičeskij analiz, Novosibirsk.

Tesnière, Lucien, 1959, Eléments de syntaxe structurale, préface de Jean Fourquet, Paris, Klincksieck (cité d'après la $2^{\circ}$ éd., 1969).

Povzetek

\section{SKLADNJA IN POMENOSLOVJE PRI TESNIÈRJU}

Strukturalna skladnja Luciena Tesnièrja vsebuje razen teorije prenosa dve glavni smeri:

1. Teorijo odvisnosti, na katero so gotovo vplivale šolske slovnice in pa doktrina Charlesa Ballyja, pojavlja pa se pri Tesnièrju močno prečiščna in sistemsko zgrajena. Tesnière se je zatekel $k$ predstavitvi $z$ drevesom, stemmo, kar je v njegovi terminologiji shema, ki odseva odvisnosti med prvinami nekega stavka. Gre torej za splošnoveljavno sredstvo za razčlenitev skladenjske zgradbe stavka, torej znotrajjezikovno analizo. Iz tega izvira pretvorbena slovnica.

2. Teorijo delovalnikov in okoliščin. Ta teorija nudi pogoje za analizo pomena stavka, se pravi, za razčlenitev pomenskih odnosov med jezikovnimi prvinami v stavku. Tudi ta teorija je bila plodna: iz nje je izšlo z ene strani novo dojemanje $v$ splošnem pomenoslovju, $z$ druge pa slovnica sklonov.

Pri Tesnièrju, tako se zdi, izhajata ti dve teoriji druga iz druge, pravzaprav pa sta med seboj neodvisni. Stemma, drevo, daje zgolj informacijo o hierarhični uvrščenosti kake besede v odnosu do drugih, ne daje pa predvideti njene skladenjske vloge, torej vloge prvega, drugega, tretjega delovalnika, ali pa okoliščne. Drevo se nanaša na vse pomensko važne prvine $v$ stavku (pri Tesnièrju samo besede, hierarhično lestvico pa si lahko zamislimo tudi za morfeme), pojem funkcionalnosti pa zadeva samo polnopomenske besede, torej tiste, ki imajo zunajjezikovno odnosnico.

Tesnière sam ostre ločnice med skladenjsko in pomensko ravnino ni postavil. Analiza odnosov med obema pa se zdi še posebej plodna smer za nadaljnje razglabljanje. 